\title{
Optical Anapole Metamaterial
}

Pin Chieh Wu ${ }^{1}$, Chun Yen Liao ${ }^{2}$, Vassili Savinov ${ }^{3}$, Tsung Lin Chung ${ }^{2}$, Wei Ting Chen ${ }^{2}$, YaoWei Huang ${ }^{1}$, Pei Ru Wu², Yi-Hao Chen ${ }^{2}$, Ai-Qun Liu ${ }^{4}$, Nikolay I. Zheludev ${ }^{3,5}$ and Din Ping Tsai ${ }^{1,2,6}$

${ }^{1}$ Research Center for Applied Sciences, Academia Sinica, Taipei 11529, Taiwan

${ }^{2}$ Department of Physics, National Taiwan University, Taipei 10617, Taiwan

${ }^{3}$ Optoelectronics Research Centre and Centre for Photonic Metamaterials, University of Southampton, Southampton SO17 1BJ, UK

${ }^{4}$ School of Electrical and Electronic Engineering, Nanyang Technological University, Singapore 639798

${ }^{5}$ TPI and Centre for Disruptive Photonic Technologies, Nanyang Technological University, Singapore 637371, Singapore

${ }^{6}$ College of Engineering, Chang Gung University, Taoyuan 33302, Taiwan

*Corresponding Author: Din Ping Tsai, E-mail: dptsai@ sinica.edu.tw 


\begin{abstract}
The toroidal dipole is a localized electromagnetic excitation independent from the familiar magnetic and electric dipoles. It corresponds to currents flowing along minor loops of a torus. Interference of radiating induced toroidal and electric dipoles leads to anapole, a non-radiating charge-current configuration. Interactions of induced toroidal dipoles with electromagnetic waves have recently been observed in artificial media at microwave, terahertz and optical frequencies. Here, we demonstrate a quasi-planar plasmonic metamaterial - a combination of dumbbell aperture and vertical split-ring resonator - that exhibits transverse toroidal moment and resonant anapole behavior in the optical part of the spectrum upon excitation with a normally incident electromagnetic wave. Our results prove experimentally that toroidal modes and anapole modes can provide distinct and physically significant contributions to the absorption and dispersion of slabs of matter in the optical part of the spectrum in conventional transmission and reflection experiments.
\end{abstract}

Keywords: toroidal dipole; anapole; toroidal metamaterial; dumbbell aperture; vertical splitring resonator. 
Static toroidal dipole was first proposed in 1957 in the context of nuclear physics. ${ }^{1-3}$ Dynamic (induced) toroidal dipole is an independent term in the family of electrodynamic multipole expansion. ${ }^{4}$ It is associated with a non-vanishing longitudinal term $\vec{r} \cdot \vec{J} \neq 0$, where $\vec{r}$ and $\vec{J}$ are position vector and current density induced by the external stimuli, respectively. Hence, toroidal dipole is naturally different from the electric and magnetic multipoles that are associated with non-vanishing induced charge density, $\rho \neq 0$, and non-zero induced transverse current density, $\vec{r} \times \vec{J} \neq 0$, respectively (see box 2 in Ref. [4]). Historically, toroidal dipolar response was neglected as a contributor to the electromagnetic response of matter because the corresponding toroidal transition matrix elements tend to be weak for atoms and small molecules. It has been proved that the near-field characteristics and electromagnetic multipoles can be artificially tailored through plasmonic meta-structures..$^{5-8}$ Few years ago, toroidal dipole excitation was for the first time experimentally observed as the dominant term in the electromagnetic response of purposely designed metamaterials ${ }^{9-11}$ in the microwave region. ${ }^{12}$ Subsequently, the toroidal resonances have also been pushed to terahertz and optical frequencies with metallic and dielectric metamaterials. ${ }^{13-17}$ The destructive interference between co-located and co-aligned toroidal and electric dipoles yield a non-radiating anapole mode,${ }^{18-20}$ which was also first discovered in microwave range ${ }^{21}$ So far most of the published results explored structures where the toroidal dipoles are generated perpendicular to the surface of the metamaterials. ${ }^{16,22-24}$ Such excitations are difficult to couple to external radiation without resorting to complex excitation geometries. Here, for the first time we experimentally demonstrate quasi-planar plasmonic metamaterials exhibiting induced transverse toroidal response and associated resonant anapole response in the optical part of the spectrum, driven by normally incident light.

The novel toroidal metamaterial reported here is a multi-layered structure containing a planar array of vertical split-ring resonators (VSRRs) suspended in a dielectric medium and 
covered with a perforated gold film. The perforations (apertures) in the gold film are dumbbellshaped and are aligned to VSRRs (see Fig. 1a). At resonance, the normally-incident plane-wave radiation, polarized along the VSRRs, shall induce toroidal dipole excitations along the same direction, as illustrated in Fig. 1a. Such excitations will be coupled to free-space radiation and will therefore directly contribute to the optical response of the metamaterial. Figure 1b shows radiation pattern of a point-like toroidal dipole superimposed with the single unit cell of the metamaterial. The radiation pattern of the toroidal dipole is identical to that of the electric dipole. Combining these two dipoles, with appropriate amplitude and phase relationship, therefore allows destructive interference, resulting in the so-called anapole mode with strong fields in the vicinity of the source and vanishingly small far-field emission, as shown in Fig. 1c. The metamaterial presented here includes a tunable parameter, the spacing between VSRRs and the perforated gold film (see Fig. 1a), that allows to tune the resonant wavelength of both toroidal dipole and non-radiating anapole mode. This parameter can be adjusted by controlling thickness of the deposited dielectric spacer. To our best knowledge, this is the first demonstration of anapole-supporting plasmonic nanostructure that offers such flexibility and reliability in optimization of sample geometry.

\section{RESULTS AND DISCUSSIONS}

To motivate the design of the metamaterial depicted in Fig. 1, we first consider the modelled response of a simpler sample that consists of only the dumbbell-perforated gold film on dielectric substrate, as shown in Fig. 2. The metamaterial is driven by horizontally polarized ( $x$-polarized in Fig. 2a) plane-wave radiation at normal incidence. The incident light induces charge oscillations at the two central edges of the apertures (green ' + ' and '-' in Fig. 2a), leading to a strong horizontal electric dipole. The charge oscillations are accompanied by counter-rotating current oscillations along the rims of the two circular holes that make up the apertures (blue circulating arrows in Fig. 2a), in turn, this leads to a pair of counter-oriented 
out-of-plane magnetic dipoles (red arrows in Fig. 2a). A pair of counter-oriented magnetic dipoles, corresponds to a superposition of equal-magnitude magnetic quadrupole and toroidal dipole $^{17}$, as shown in Fig $2 \mathrm{~b}$. Therefore, the response of the metamaterial is dominated by a combination of electric and toroidal dipoles as well as magnetic quadrupole. Figure $2 \mathrm{c}$ shows the calculated transmission and reflection spectra of dumbbell aperture metamaterial (horizontal polarization) which we decomposed into contributions of individual multipoles. ${ }^{25}$ Here, the origin position in the $x y$-plane is chosen based on the symmetry (at the center of the unit cell), whilst the origin position along $z$-axis is assumed to be at the top of the metamaterial (just above the gold film). The main objective of the multipole decomposition is to represent a localized electromagnetic excitation in a way that is irreducible under 3-dimensional (3D) rotations. The source of electromagnetic excitation is the charge-current density, which is a 4dimensional vector field subject to one constraint in the form of charge conservation equation. In practice, this means that current density can be regarded as a completely arbitrary $3 \mathrm{D}$ vector field and the charge density is then obtained from it. To provide full multipole decomposition of an arbitrary vector field one needs three multipole families, that is, electric, magnetic, and toroidal dipoles. ${ }^{26}$ In Fig. 2d, the strength of multipole excitations is compared by considering power scattered over the full $4 \pi$ solid angle. ${ }^{12,}{ }^{26}$ As expected from mode analysis in Figs. 2a and $2 b$, the main contributors to metamaterial response are the electric dipole and a superposition of closely matched toroidal dipole and magnetic quadrupole. The validity of multipole decomposition shown in Fig. $2 \mathrm{~d}$ is verified by adding all multipole fields to reproduce the metamaterial response (see Fig. 2c).

The dumbbell aperture alone shown in Fig. 2 cannot produce dominant toroidal dipole response. However, combining the dumbbell apertures with VSRR, ${ }^{27,} 28$ i.e. changing the metamaterial design from Fig. 2a to Fig. 1a, breaks the symmetry, resulting in enhanced toroidal dipole and suppressed magnetic quadrupole. Indeed, VSRRs generate strong magnetic 
dipolar response under normal illumination when the incident electric field is parallel to the opening gap. ${ }^{29-31}$ Combining the magnetic dipole induced in VSRR with the two magnetic dipoles due to counter-rotating currents in dumbbell aperture, results in the predominantly toroidal excitation of the merged structure (see inset in Fig. 3a). The transmission and reflection spectra of the combined metamaterial (VSRR and dumbbell apertures) are shown in Figs. 3a and $3 \mathrm{~b}$ as a function of radiation wavelength and separation between the VSRRs and the perforated gold film (gap size, see Fig. 1a). Figures 3c and 3d show the distribution of magnetic field induced in the unit cell of the combined metamaterial at toroidal resonance, confirming the picture discussed above. Indeed, at resonance the response of the dumbbell aperture results in two counter-oriented out-of-plane magnetic dipoles, whilst the contribution of the VSRR corresponds to $y$-polarized magnetic dipole. The net effect is a tight loop of oscillating magnetic field (Fig. 3d), a hallmark of toroidal dipole response. An important feature of metamaterial's resonant response here is that the mode (i.e. electromagnetic field) associated with strong toroidal dipole response, extends beyond the physical boundaries of the metamaterial (Fig. 3d). This is of great practical significance, since one can exploit this 'leaking' field for applications such as in sensing. By contrast, in dielectric toroidal/anapole metamaterials reported previously, ${ }^{16,24,32-34}$ the field remains hidden within the bulk of the dielectric, greatly reducing the practical utility of these devices. It is worth mentioning that in case of vertical polarization of incident field (along $y$-axis in Fig 1a) electric quadrupole and magnetic dipole dominate the radiated fields (see Supplementary).

Figure 4a shows the power scattered by individual multipoles that form the response of the planar toroidal metamaterial (see Fig. 3). Toroidal dipole emission, which is about 85.1 and 2.5 times respectively stronger than that of electric and magnetic dipoles, clearly dominates in the window around $1350 \mathrm{~nm}$ (olive region in Fig. 4a). Next, we consider interference between electric and toroidal dipoles. The scattered powers of these two multipoles are equal at around 
$1400 \mathrm{~nm}$ (dashed line in Fig. 4a) while all other multipole contributions are relatively small. The phase difference between emissions of toroidal and electric dipoles, presented in Fig $4 \mathrm{a}$ (bottom graph), shows that at that wavelength, the two dipoles interfere destructively, resulting in a single resonant dip in the scattering power spectrum [bottom panel in Fig. 4(a)]. This is the so-called anapole mode, which is manifested as simultaneous enhancement of the near-field and suppression of the far-field. The suppression of the far-field scattering is also verified by calculating the far-field scattering cross-section (and extinction spectra, see Supplementary) from a single unit structure, as shown in Fig. $4 \mathrm{~b}$. The spectral position of anapole mode of the planar toroidal metamaterial depends on the separation between VSRRs and the perforated gold film (gap size; see Fig. 1a) as shown in Fig. 4c.

The relationship between gap size and strength of the toroidal dipole response in planar toroidal metamaterial is further investigated in Fig. 5. Figure 5a shows the extent to which toroidal dipole scattering can dominate over other multipoles as a function of gap size, revealing that dominant toroidal dipole response is only possible when VSRR is close to the perforated gold film. At close proximity, the effect of VSRR is to contribute to creating tight oscillating loop of magnetic field (Fig. 3d), which leads to toroidal dipole excitation. When the gap size is increased the oscillations of VSRR start to fall behind those of dumbbell aperture (a phase difference appears), which favors magnetic quadrupole. Hence at gap size $20 \mathrm{~nm}$ the toroidal dipole emerges as the dominant multipole at wavelengths around $1270 \mathrm{~nm}$, whereas at gap size $635 \mathrm{~nm}$ it is the magnetic quadrupole that dominates at around $1900 \mathrm{~nm}$ (see Fig. $5 \mathrm{~b}$ and the Supplementary movie).

Finally, we experimentally examine the optical properties of the proposed planar toroidal metamaterials. Figure 6a shows the top view SEM image of the fabricated sample. The integrity and accuracy of the multilayer toroidal metamaterials is confirmed by cutting a cross-section in the fabricated sample using focused ion beam (FIB) milling. Figure $6 \mathrm{~b}$ displays an oblique 
SEM image of metamaterial cross-section, with $52^{\circ}$ tilt, showing an accurate alignment between different steps of e-beam lithography process. The measured spectra for the toroidal metamaterials with $50 \mathrm{~nm}$ gap size (as defined in Fig 1a) are shown in Fig. 6c. Metamaterial resonance occurs at $1389 \mathrm{~nm}$, in good agreement with modeling results shown in Fig. 3a. The slight shift and broader resonance in the experimental measurements arise as a result of inevitable variations in metamaterial dimensions and roughness of the fabricated sample. The dependence of material losses on the anapole mode excitation is investigated in the Supplementary. On the other hand, one can clearly observe a minimum far-field scattering intensity (defined as the reflection plus transmission) at the wavelength of anapole mode in the experimental spectra, which agrees with the numerical prediction shown in Fig. 4b.

The precision-engineered electromagnetic nanostructure presented here enables the excitation of toroidal dipole and anapole modes at the wavelength of choice. The suppression of radiation losses offered by multipole interference in anapole modes could find many applications. For example, the high energy confinement at the wavelength of non-radiating anapole mode enable significantly enhancing the nonlinear response. ${ }^{35,} 36$ The great field confinement, which leads to a strong gradient force around the plasmonic structures, could be applied in optical trapping. ${ }^{37}$ The optical response with high quality-factor can further benefit the development of novel nanophotonic light sources, including lasing spasers etc. ${ }^{38,39}$ In our design, with plasmonic mode being accessible in free space (close to the sample), it is also applicable to sensing applications such as surface enhanced Raman scattering (SERS). Development of toroidal spectroscopy, which is highly desirable to observe and analyze the toroidal resonances in natural matters, is also feasible due to the efficient couple between anapole metamaterials and interested toroidal molecules. Furthermore, localized toroidal excitations, such as those demonstrated here, are a natural starting point for experimental study of the exotic topological configurations known as electromagnetic focused doughnut pulses. ${ }^{4}$ 


\section{CONCLUSIONS}

In summary, we have designed, analyzed and fabricated the first planar plasmonic metamaterial, which supports transverse toroidal dipole and anapole excitations that dominate metamaterial's resonant response in the optical part of the spectrum. All significant properties of the anapole metamaterial can be tuned through a single well-defined geometrical parameter, which can be controlled to a great precision during fabrication. It benefits the design of optical metamaterials with desired multipole response while the others are suppressed on purpose. Our technology offers fine control over the radiation loss, and near-field enhancement in nanophotonics. This work could therefore have immediate applications in sensing, nonlinear optics and optomechanics.

\section{METHODS}

\section{Fabrication:}

A ZEP520A layer is spin coated at $4000 \mathrm{rpm}$ on a fused silica substrate and then bake on a hot plate for 5 minutes at $180^{\circ} \mathrm{C}$. Subsequently, an Espacer layer is spin coated at $1500 \mathrm{rpm}$ on the ZEP520A layer. Espacer is an organic polymer with high conductivity to reduce the positional error during the e-beam exposure process. An e-beam writing system at the acceleration voltage of $100 \mathrm{keV}$ with 30 pico-ampere of current is used. The base rod of VSRR is defined by the first e-beam exposure and lift-off process. The two prongs of VSRR are fabricated by the second e-beam exposure and lift-off process. In our case, we use spin on glass (SOG) for the spacer layer to isolate the VSRR and dumbbell-shaped structure. SOG layer is subsequently spin-coated at $3000 \mathrm{rpm}$ on fused silica substrate with fabricated VSRR array and then bake on a hot plate for $3 \mathrm{~min}$ at $200{ }^{\circ} \mathrm{C}$. The Reactive Ion Etching (SAMCO RIE-10N, reaction gas is $\mathrm{CF}_{4}$, flow rate is $50 \mathrm{sccm}$, pressure is $0.78 \mathrm{kpa}$, $\mathrm{RF}$ power is $70 \mathrm{~W}$, and etching time is $1 \mathrm{~min}$ ) is used to attain the appropriate thickness $(50 \mathrm{~nm})$ of SOG layer we need. Then, we use RF sputter to deposit $30 \mathrm{~nm}$ gold films on sample, and spin coating ZEP520A over the gold film. 
Using the third e-beam exposure to process the dumbbell-shaped holes in the same area, which will be fabricated after RIE etching (ANELVA DEM-451T, reaction gas: Ar, flow rate: $20 \mathrm{sccm}$, pressure: 0.02 mbar, RF power: $100 \mathrm{~W}$, and etching time: $3 \mathrm{~min}$ ). Here, the photoresist (ZEP520A) is used as the anti-etch layer.

\section{Measurement and simulation}

The measurement data is carried out by Bruker VERTEX 70 Fourier-transform infrared spectrometer equipped with Bruker HYPERION 2000 infrared microscope $(15 \times$ Cassegrain objective, numerical aperture NA $=0.4$, near-infrared polarizer, and an InGaAs and MCT detector). An iris is used to collect the incident light to a square area of about $150 \times 150 \mu \mathrm{m}^{2}$. The reflectance spectrum is normalized by the gold mirror.

The simulated spectra are calculated by the numerical simulation software (COMSOL Multiphysics) based on finite-element method (FEM). The refractive index of the fused silica substrate and SOG layer are set as 1.4584 and 1.41, respectively. The permittivity of gold is described by the Lorentz-Drude model with a damping constant of $0.07 \mathrm{eV}$ and a plasma frequency of $8.997 \mathrm{eV}$. For the simulation of array structures, periodic boundary condition is used along $x$ and $y$ directions. The incident wave is defined as either an $x$ - or $y$-polarized plane wave. By calculating the scattering parameters (S-parameters), we can directly obtain the optical reflection as well as transmission spectra.

\section{Supporting Information}

The Supporting Information is available free of charge on the ACS Publications website at DOI: XXX. Section 1: Electromagnetic multipole expansion; Section 2: Optical response of VSRR; Section 3: Optical spectra with electromagnetic multipole expansion; Section 4: Extinction spectra in the optical anapole metamaterials; Section 5: Optical response of toroidal 
metamaterials with y-polarized illumination; Section 6: Independence of electric and toroidal dipoles; Section 7: Loss-dependence of anapole mode excitation.

\section{ACKNOWLEDGMENTS}

The authors acknowledge financial support from Ministry of Science and Technology, Taiwan (Grant No. MOST-106-2745-M-002-003-ASP) and Academia Sinica (Grant No. AS-103-TPA06), the UK Engineering and Physical Sciences Research Council (Grant EP/M009122/1) and the Singapore Ministry of Education (Grant MOE2011-T3-1-005). They are also grateful to National Center for Theoretical Sciences, NEMS Research Center of National Taiwan University, National Center for High-Performance Computing, Taiwan, and Research Center for Applied Sciences, Academia Sinica, Taiwan for their supports. 


\section{References}

1. Esposito, S. Transition from Majorana to Weyl Fermions and Anapole Moments. EPL (Europhysics Letters) 2013, 102, 17006.

2. Dubovik, V. M.; Martsenyuk, M. A.; Saha, B. Material Equations for Electromagnetism with Toroidal Polarizations. Phys. Rev. E 2000, 61, 7087-7097.

3. Dubovik, V. M.; Tugushev, V. V. Toroid Moments in Electrodynamics and Solid-state Physics. Phys. Rep. 1990, 187, 145-202.

4. Papasimakis, N.; Fedotov, V. A.; Savinov, V.; Raybould, T. A.; Zheludev, N. I. Electromagnetic Toroidal Excitations in Matter and Free Space. Nat. Mater. 2016, 15, 263271.

5. Tseng, M. L.; Yang, J.; Semmlinger, M.; Zhang, C.; Nordlander, P.; Halas, N. J. TwoDimensional Active Tuning of an Aluminum Plasmonic Array for Full-Spectrum Response. Nano Lett. 2017, 17, 6034-6039.

6. Wu, P. C.; Papasimakis, N.; Tsai, D. P. Self-Affine Graphene Metasurfaces for Tunable Broadband Absorption. Phys. Rev. Applied 2016, 6, 044019.

7. Zhang, R.; Bursi, L.; Cox, J. D.; Cui, Y.; Krauter, C. M.; Alabastri, A.; Manjavacas, A.; Calzolari, A.; Corni, S.; Molinari, E.; Carter, E. A.; García de Abajo, F. J.; Zhang, H.; Nordlander, P. How To Identify Plasmons from the Optical Response of Nanostructures. ACS Nano 2017, 11, 7321-7335.

8. König, M.; Rahmani, M.; Zhang, L.; Lei, D. Y.; Roschuk, T. R.; Giannini, V.; Qiu, C.-W.; Hong, M.; Schlücker, S.; Maier, S. A. Unveiling the Correlation between Nanometer-Thick Molecular Monolayer Sensitivity and Near-Field Enhancement and Localization in Coupled Plasmonic Oligomers. ACS Nano 2014, 8, 9188-9198.

9. Zhang, L.; Mei, S.; Huang, K.; Qiu, C.-W. Advances in Full Control of Electromagnetic Waves with Metasurfaces. Adv. Opt. Mater. 2016, 4, 818-833.

10. Kabashin, A. V.; Evans, P.; Pastkovsky, S.; Hendren, W.; Wurtz, G. A.; Atkinson, R.; Pollard, R.; Podolskiy, V. A.; Zayats, A. V. Plasmonic Nanorod Metamaterials for Biosensing. Nat. Mater. 2009, 8, 867-871.

11. Jahani, S.; Jacob, Z. All-Dielectric Metamaterials. Nat. Nano. 2016, 11, 23-36.

12. Kaelberer, T.; Fedotov, V. A.; Papasimakis, N.; Tsai, D. P.; Zheludev, N. I. Toroidal Dipolar Response in a Metamaterial. Science 2010, 330, 1510-1512.

13. Basharin, A. A.; Kafesaki, M.; Economou, E. N.; Soukoulis, C. M.; Fedotov, V. A.; Savinov, V.; Zheludev, N. I. Dielectric Metamaterials with Toroidal Dipolar Response. Phys. Rev. X 2015, 5, 011036.

14. Liu, Z.; Du, S.; Cui, A.; Li, Z.; Fan, Y.; Chen, S.; Li, W.; Li, J.; Gu, C. High-Quality-Factor Mid-Infrared Toroidal Excitation in Folded 3D Metamaterials. Adv. Mater. 2017, 19, 1606298.

15. Huang, Y.-W.; Chen, W. T.; Wu, P. C.; Fedotov, V.; Savinov, V.; Ho, Y. Z.; Chau, Y.-F.; Zheludev, N. I.; Tsai, D. P. Design of Plasmonic Toroidal Metamaterials at Optical Frequencies. Opt. Express 2012, 20, 1760-1768.

16. Ögüt, B.; Talebi, N.; Vogelgesang, R.; Sigle, W.; van Aken, P. A. Toroidal Plasmonic Eigenmodes in Oligomer Nanocavities for the Visible. Nano Lett. 2012, 12, 5239-5244.

17. Gupta, M.; Savinov, V.; Xu, N.; Cong, L.; Dayal, G.; Wang, S.; Zhang, W.; Zheludev, N. I.; Singh, R. Sharp Toroidal Resonances in Planar Terahertz Metasurfaces. Adv. Mater. 2016, 28, 8206-8211.

18. Wei, L.; Xi, Z.; Bhattacharya, N.; Urbach, H. P. Excitation of the Radiationless Anapole Mode. Optica 2016, 3, 799-802.

19. Miroshnichenko, A. E.; Evlyukhin, A. B.; Yu, Y. F.; Bakker, R. M.; Chipouline, A.; Kuznetsov, A. I.; Luk/'yanchuk, B.; Chichkov, B. N.; Kivshar, Y. S. Nonradiating Anapole Modes in Dielectric Nanoparticles. Nat. Commun. 2015, 6, 8069. 
20. Nemkov, N. A.; Basharin, A. A.; Fedotov, V. A. Nonradiating Sources, Dynamic Anapole, and Aharonov-Bohm Effect. Phys. Rev. B 2017, 95, 165134.

21. Fedotov, V. A.; Rogacheva, A. V.; Savinov, V.; Tsai, D. P.; Zheludev, N. I. Resonant Transparency and Non-Trivial Non-Radiating Excitations in Toroidal Metamaterials. Sci. Rep. 2013, 3, 2967.

22. Li, J.; Zhang, Y.; Jin, R.; Wang, Q.; Chen, Q.; Dong, Z. Excitation of Plasmon Toroidal Mode at Optical Frequencies by Angle-Resolved Reflection. Opt. Lett. 2014, 39, 66836686.

23. Dong, Z.-G.; Zhu, J.; Yin, X.; Li, J.; Lu, C.; Zhang, X. All-optical Hall Effect by the Dynamic Toroidal Moment in a Cavity-based Metamaterial. Phys. Rev. B 2013, 87, 245429.

24. Dong, Z.-G.; Zhu, J.; Rho, J.; Li, J.-Q.; Lu, C.; Yin, X.; Zhang, X. Optical Toroidal Dipolar Response by an Asymmetric Double-Bar Metamaterial. Appl. Phys. Lett. 2012, 101, 144105-144105-4.

25. Savinov, V.; Fedotov, V. A.; Zheludev, N. I. Toroidal Dipolar Excitation and Macroscopic Electromagnetic Properties of Metamaterials. Phys. Rev. B 2014, 89, 205112.

26. Radescu, E. E.; Vaman, G. Exact Calculation of the Angular Momentum Loss, Recoil Force, and Radiation Intensity for an Arbitrary Source in terms of Electric, Magnetic, and Toroid Multipoles. Phys. Rev. E 2002, 65, 046609.

27. Wu, P. C.; Chen, W. T.; Yang, K.-Y.; Hsiao C. T.; Sun, G.; Liu A. Q.; Zheludev, N. I.; Tsai, D. P. Magnetic Plasmon Induced Transparency in Three-Dimensional Metamolecules. Nanophotonics 2012, 1, 131-138.

28. Wu, P. C.; Sun, G.; Chen, W. T.; Yang, K.-Y.; Huang, Y.-W.; Chen, Y.-H.; Huang, H. L.; Hsu, W.-L.; Chiang, H. P.; Tsai, D. P. Vertical Split-Ring Resonator based Nanoplasmonic Sensor. Appl. Phys. Lett. 2014, 105, 033105.

29. Wu, P. C.; Liao, C. Y.; Chen, J.-W.; Tsai, D. P. Isotropic Absorption and Sensor of Vertical Split-Ring Resonator. Adv. Opt. Mater. 2017, 5, 161104.

30. Wu, P. C.; Hsu, W.-L.; Chen, W. T.; Huang, Y.-W.; Liao, C. Y.; Liu, A. Q.; Zheludev, N. I.; Sun, G.; Tsai, D. P. Plasmon Coupling in Vertical Split-Ring Resonator Metamolecules. Sci. Rep. 2015, 5, 9726.

31. Fan, K.; Strikwerda, A. C.; Zhang, X.; Averitt, R. D. Three-Dimensional Broadband Tunable Terahertz Metamaterials. Phys. Rev. B 2013, 87, 161104.

32. Liu, W.; Zhang, J.; Lei, B.; Hu, H.; Miroshnichenko, A. E. Invisible Nanowires with Interfering Electric and Toroidal Dipoles. Opt. Lett. 2015, 40, 2293-2296.

33. Guo, L.; Li, M.; Ye, Q.; Xiao, B.; Yang, H. Electric Toroidal Dipole Response in SplitRing Resonator Metamaterials. The European Physical Journal B - Condensed Matter and Complex Systems 2012, 85, 1-5.

34. Fan, Y.; Wei, Z.; Li, H.; Chen, H.; Soukoulis, C. M. Low-Loss and High-Q Planar Metamaterial with Toroidal Moment. Phys. Rev. B 2013, 87, 115417.

35. Grinblat, G.; Li, Y.; Nielsen, M. P.; Oulton, R. F.; Maier, S. A. Efficient Third Harmonic Generation and Nonlinear Subwavelength Imaging at a Higher-Order Anapole Mode in a Single Germanium Nanodisk. ACS Nano 2017, 11, 953-960.

36. Grinblat, G.; Li, Y.; Nielsen, M. P.; Oulton, R. F.; Maier, S. A. Enhanced Third Harmonic Generation in Single Germanium Nanodisks Excited at the Anapole Mode. Nano Lett. 2016, 16, 4635-4640.

37. Wang, R.; Dal Negro, L. Engineering Non-radiative Anapole Modes for Broadband Absorption Enhancement of Light. Opt. Exp. 2016, 24, 19048-19062.

38. Huang, Y.-W.; Chen, W. T.; Wu, P. C.; Fedotov, V. A.; Zheludev, N. I.; Tsai, D. P. Toroidal Lasing Spaser. Sci. Rep. 2013, 3, 1237.

39. Totero Gongora, J. S.; Miroshnichenko, A. E.; Kivshar, Y. S.; Fratalocchi, A. Anapole Nanolasers for Mode-Locking and Ultrafast Pulse Generation. Nat. Commun. 2017, 8, 
15535. 


\section{Figure captions}

Figure 1. (a) Schematic for toroidal metamaterial, which consists of gold film perforated by dumbbell-shaped apertures, situated above a planar array of VSRRs suspended in a dielectric spacer layer. At resonance metamaterial supports toroidal dipole excitations. The color arrows and the streamlines show the orientation of induced toroidal dipoles and the corresponding magnetic field distribution, respectively. The response of the metamaterial can be tuned by changing the gap size $g$. (b) Radiation pattern of a point-like toroidal dipole superimposed with a single metamaterial unit cell and a schematic depiction of the toroidal dipole excitation (pink torus and the blue circulating magnetic field). Inset shows the orientation of toroidal dipole that corresponds to radiation pattern in the main figure. (c) Non-radiating anapole mode induced when the co-aligned electric and toroidal dipoles radiate is in anti-phase (inset). The charges of electric dipole are shown by red spheres. The color mesh in (b) and (c) represents radiation intensity. The radiation intensity in (c) is much weaker than that of (b) because of the destructive interference between electric and toroidal dipoles.

Figure 2. Electromagnetic response of dumbbell-shaped apertures. (a) Left: Schematic for the dumbbell-shaped aperture. The feature sizes of metallic structures: $P_{x}=400 \mathrm{~nm}, P_{y}=850 \mathrm{~nm}$, $R=130 \mathrm{~nm}, D_{x}=50 \mathrm{~nm}, D_{y}=60 \mathrm{~nm}$. Right: orientation of incident field and the 30-nm-thick dumbbell-shaped aperture. (b) Representation of magnetic quadrupole and toroidal dipole in terms of magnetic dipoles. (c) Transmission and reflection spectra of metamaterial from direct calculation (solid lines) and from summing individual multipole contributions (stars). (d) Calculated scattered power for individual electromagnetic multipoles induced in metamaterial (unit cell) by the incident radiation. $p$ : electric dipole, $m$ : magnetic dipole, $Q^{(e)}$ : electric quadrupole, $Q^{(m)}$ : magnetic quadrupole, $T$ : toroidal dipole. 
Figure 3. Electromagnetic response of planar toroidal metamaterial. (a) Reflection and (b) transmission spectra for toroidal metamaterials as a function of gap size (defined in Fig. 1a). Cyan and purple dots indicate the reflection dip and transmission peak computed by first decomposing the metamaterial response into contributions of individual multipoles, and then adding the multipole fields together to recover the net response. Inset: Schematic for the toroidal metamaterial. The red arrows and the cyan circulating arrows indicate the induced magnetic dipoles and surface currents, respectively. The distribution of induced magnetic field and electromagnetic energy density in the vicinity of metamaterial unit cell, at toroidal dipole resonance, are shown in (c) and (d), respectively. The gap size for the structure is $50 \mathrm{~nm}$. Purple lines indicate the configuration of magnetic field. Cyan arrows present the direction of induced magnetic dipoles. Inset in (c): Schematic for the observation plane (marked as a semitransparent purple plane) of Figs. 3(c) and 3(d).

Figure 4. Multipole response of plasmonic toroidal metamaterial. (a) (Top) Scattering power of electromagnetic multipoles when gap size is $50 \mathrm{~nm}$ and (bottom) the average scattering power (blue curve) and phase difference (magenta dots) from the addition of electric fields from electric and toroidal dipoles. The olive area and dark-red dashed line denote the resonance region and wavelength of toroidal dipole and anapole mode, respectively. (b) Far-field scattering cross-section. It is defined as the ratio between powers of the radiation scattered by a single unit cell of metamaterial and incident radiation. (c) The spectral position of anapole mode as a function of gap size (defined in Fig. 1a.

Figure 5. Electromagnetic multipole evolution with different gap sizes. (a) Scattering power difference between toroidal dipole and other electromagnetic multipoles at the dip position of the electric dipole. The area with purple background indicates the region where toroidal dipolar 
response can dominate the spectrum (at a certain resonant wavelength). (b) Calculated scattering power for the five leading electromagnetic multipoles when the gap size is $20 \mathrm{~nm}$ (top panel), $420 \mathrm{~nm}$ (middle panel) and $635 \mathrm{~nm}$ (bottom panel).

Figure 6. (a) Top and (b) tilted views of SEM images of fabricated sample with 50-nm gap size. Scale bars: $500 \mathrm{~nm}$. (c) Measured far-field spectra of the plasmonic toroidal metamaterials under $x$-polarized illumination (compare to Fig. 1a). The dark-red dashed line represents the resonant wavelength of anapole mode from numerical predication. 

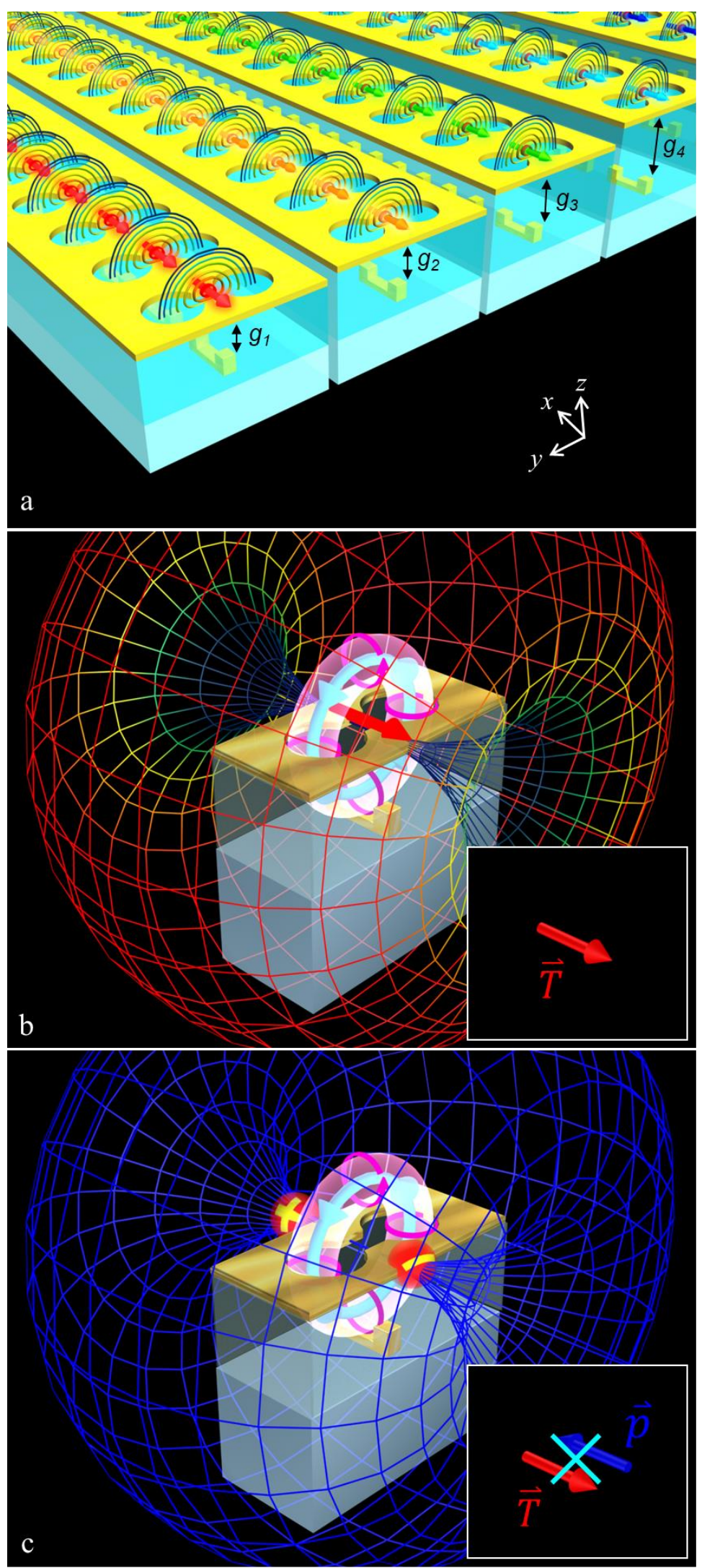

Min.

Max.

Figure 1 
a

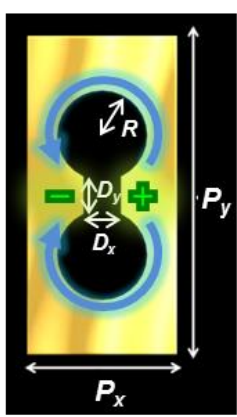

c
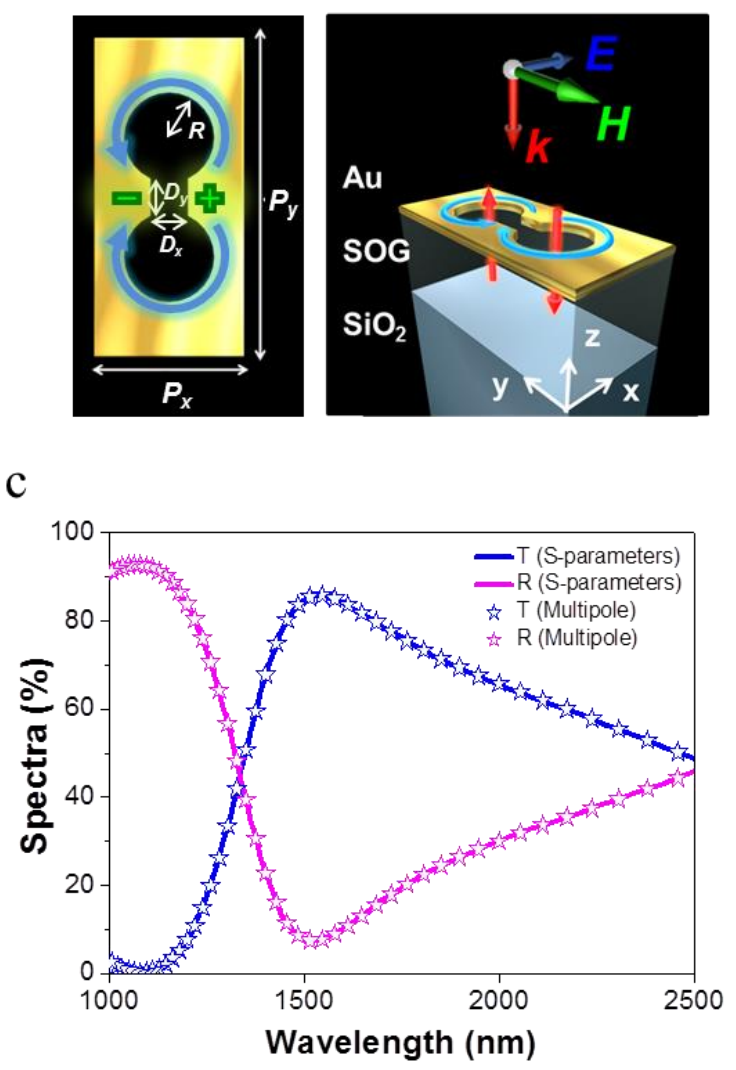

b

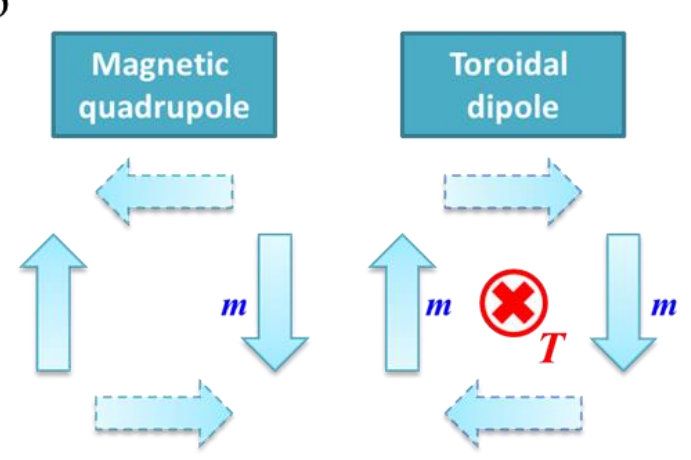

d

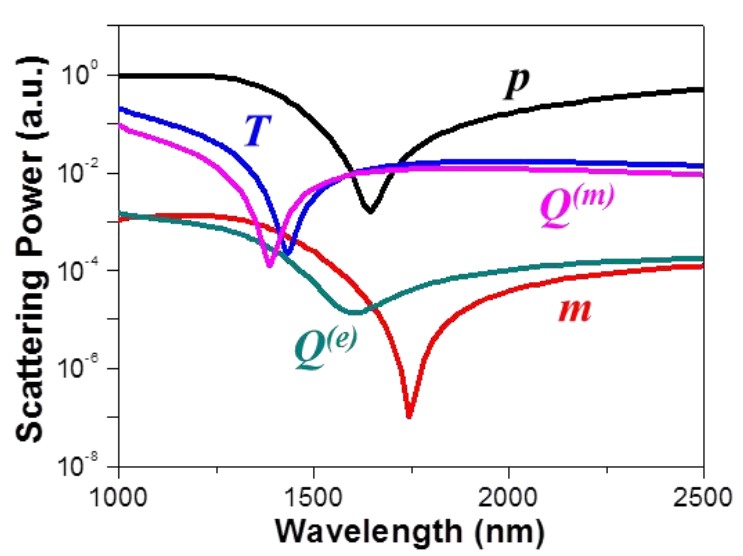

Figure 2 
a

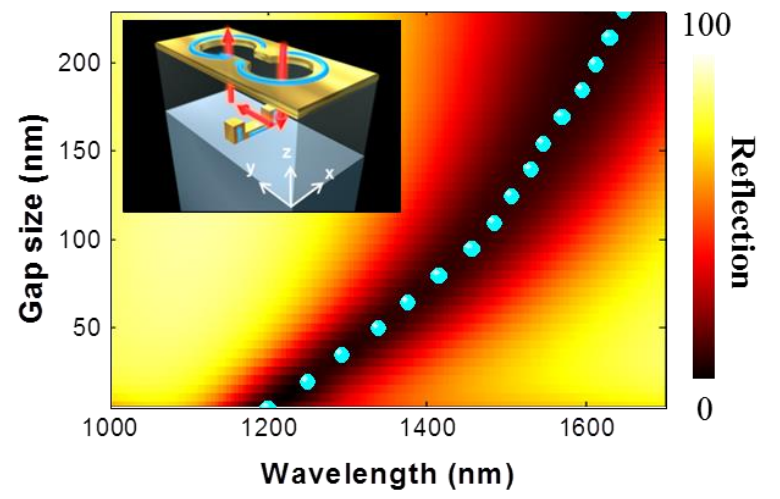

c
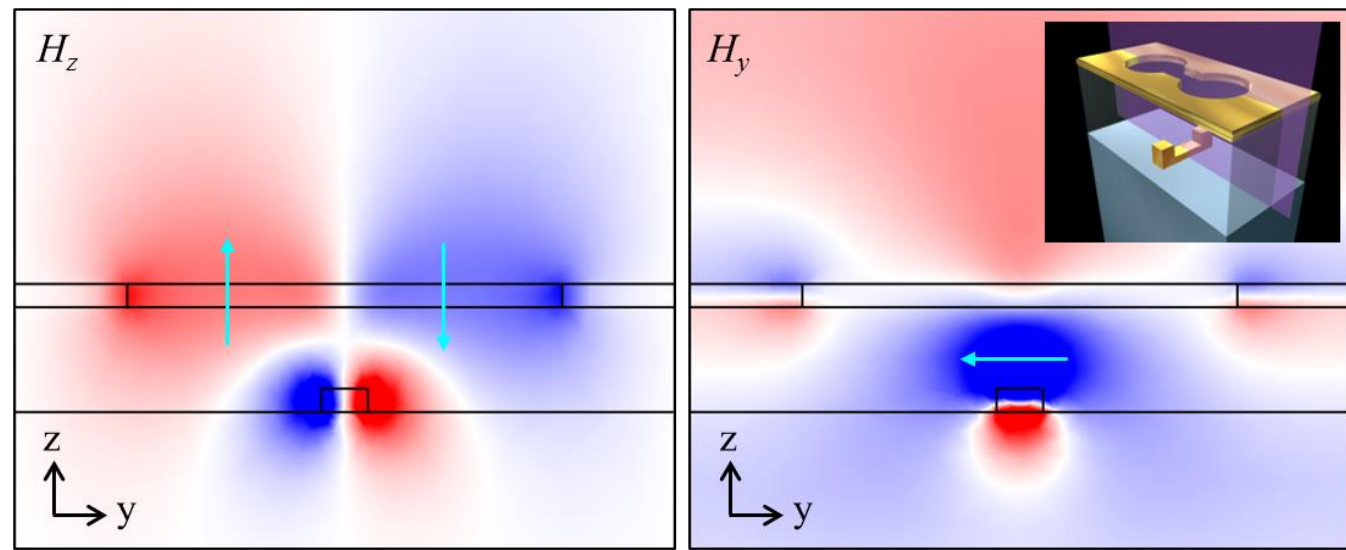

Max.

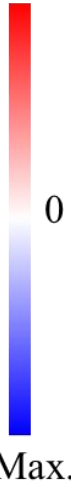

d

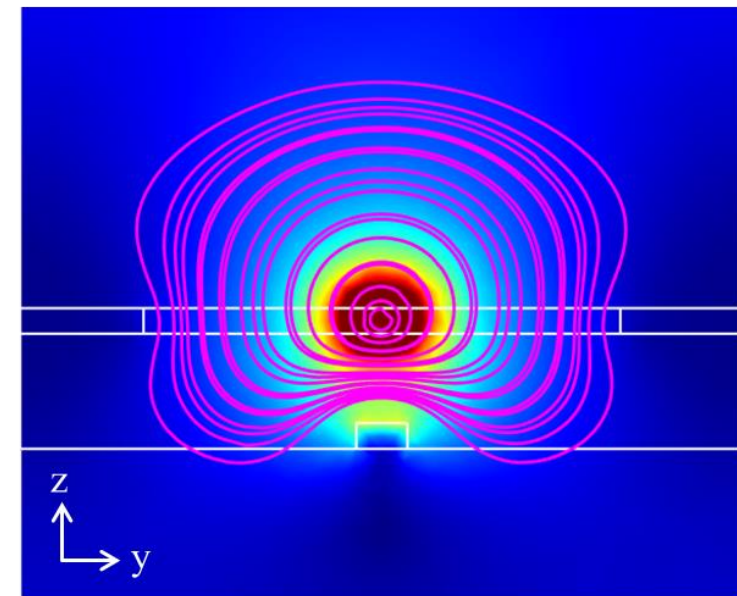

Max.

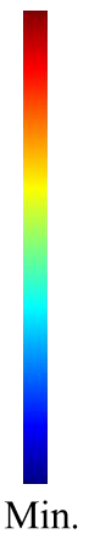

Figure 3 


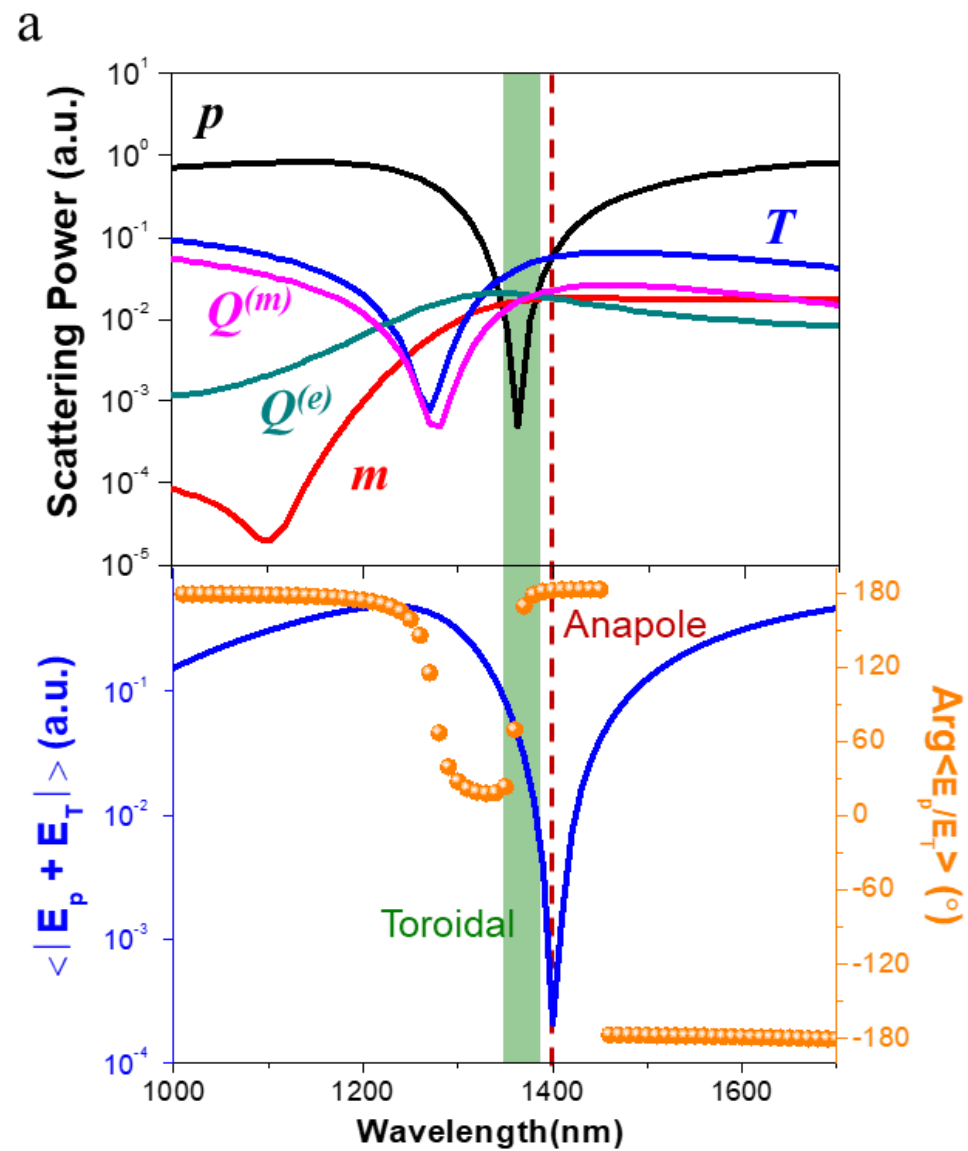

b

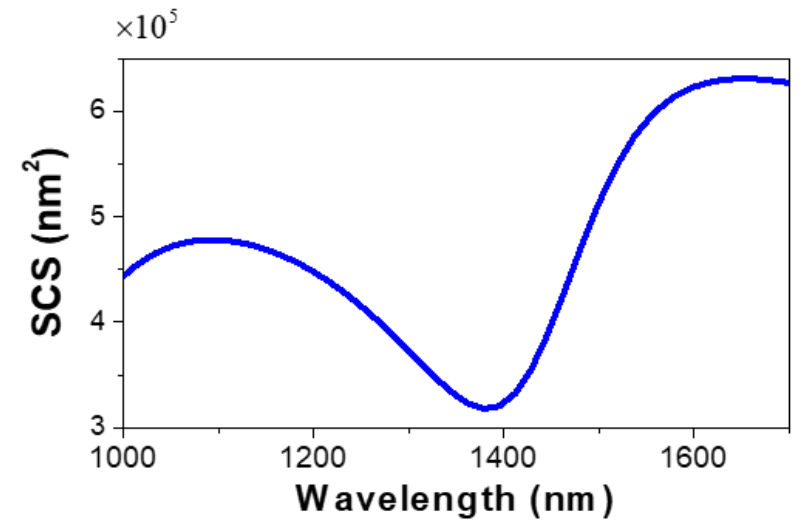

c

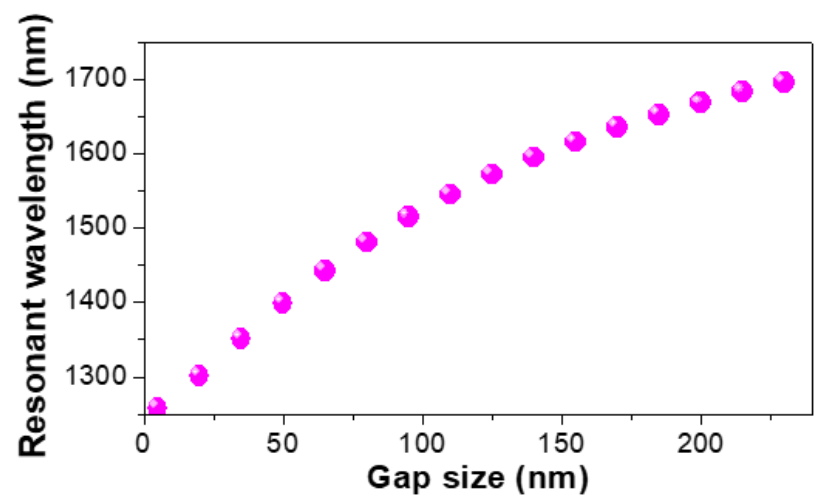

Figure 4 


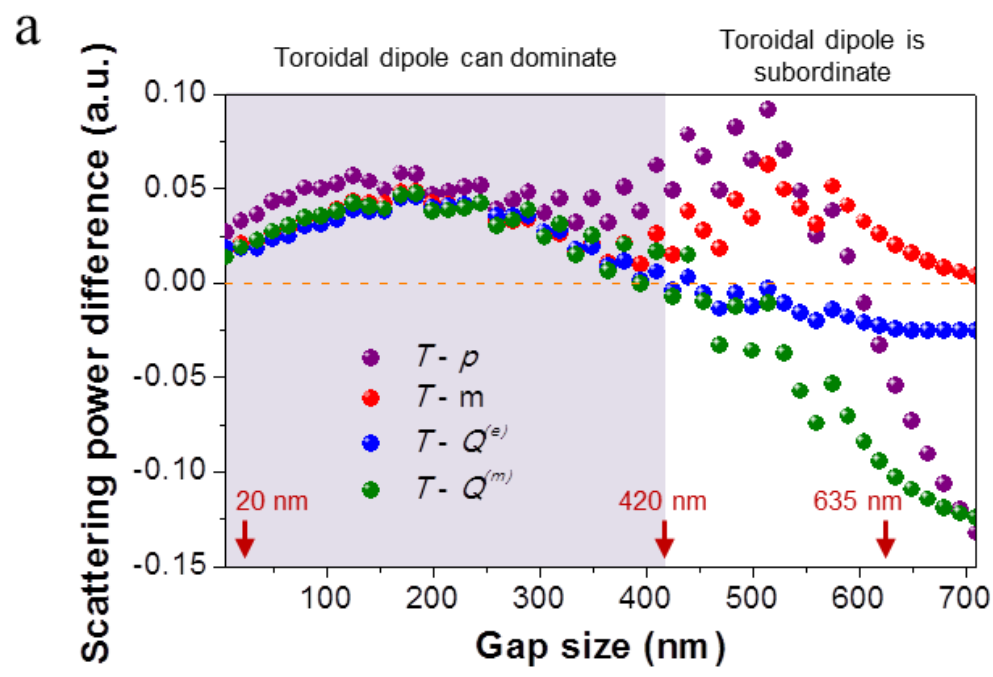

b

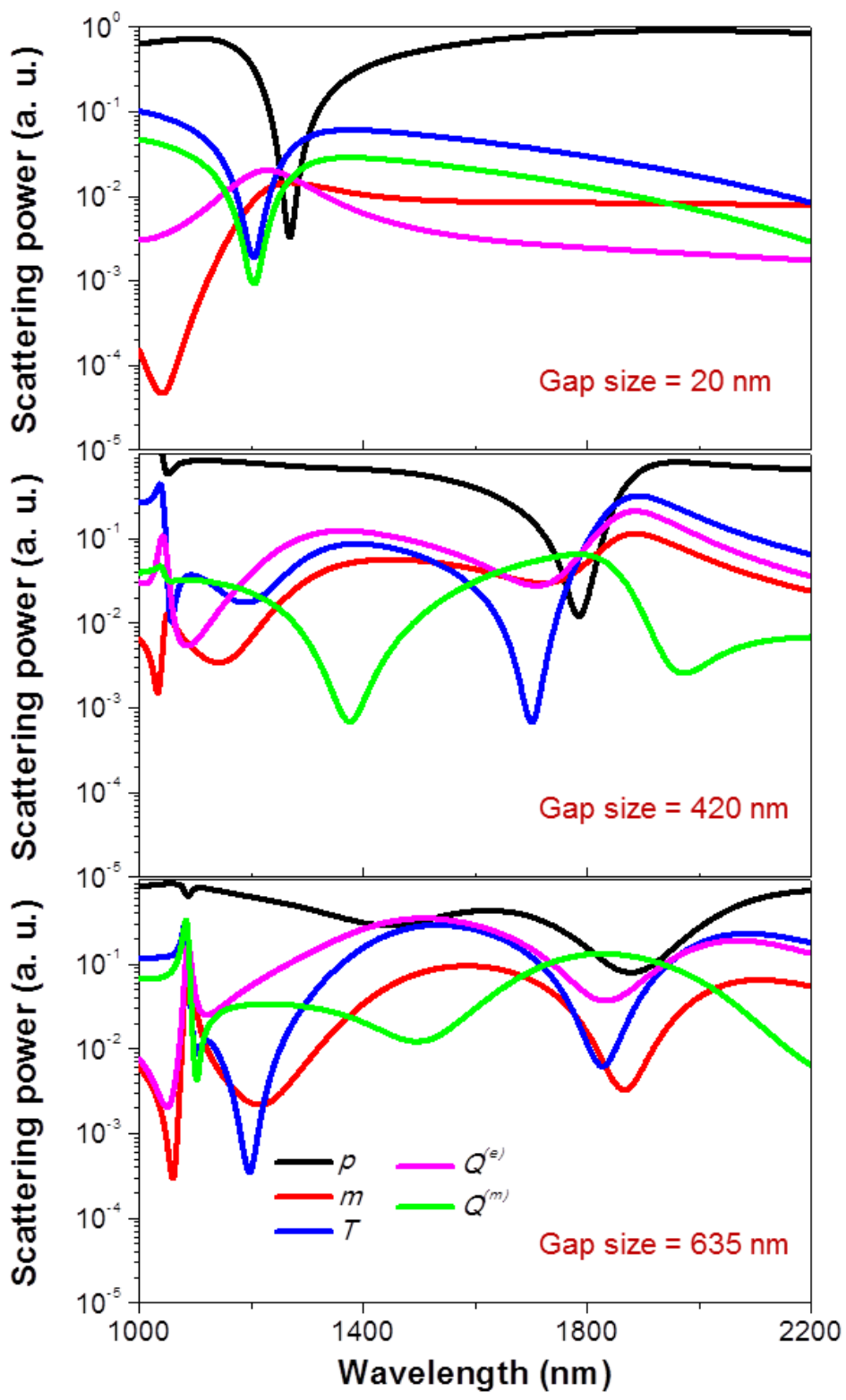

Figure 5 
a

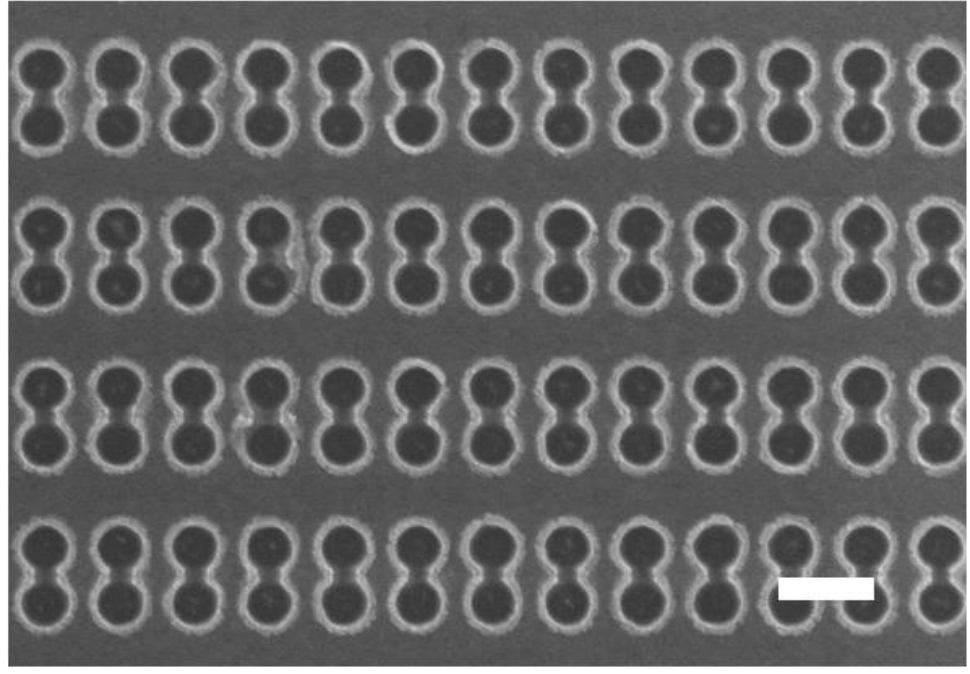

b
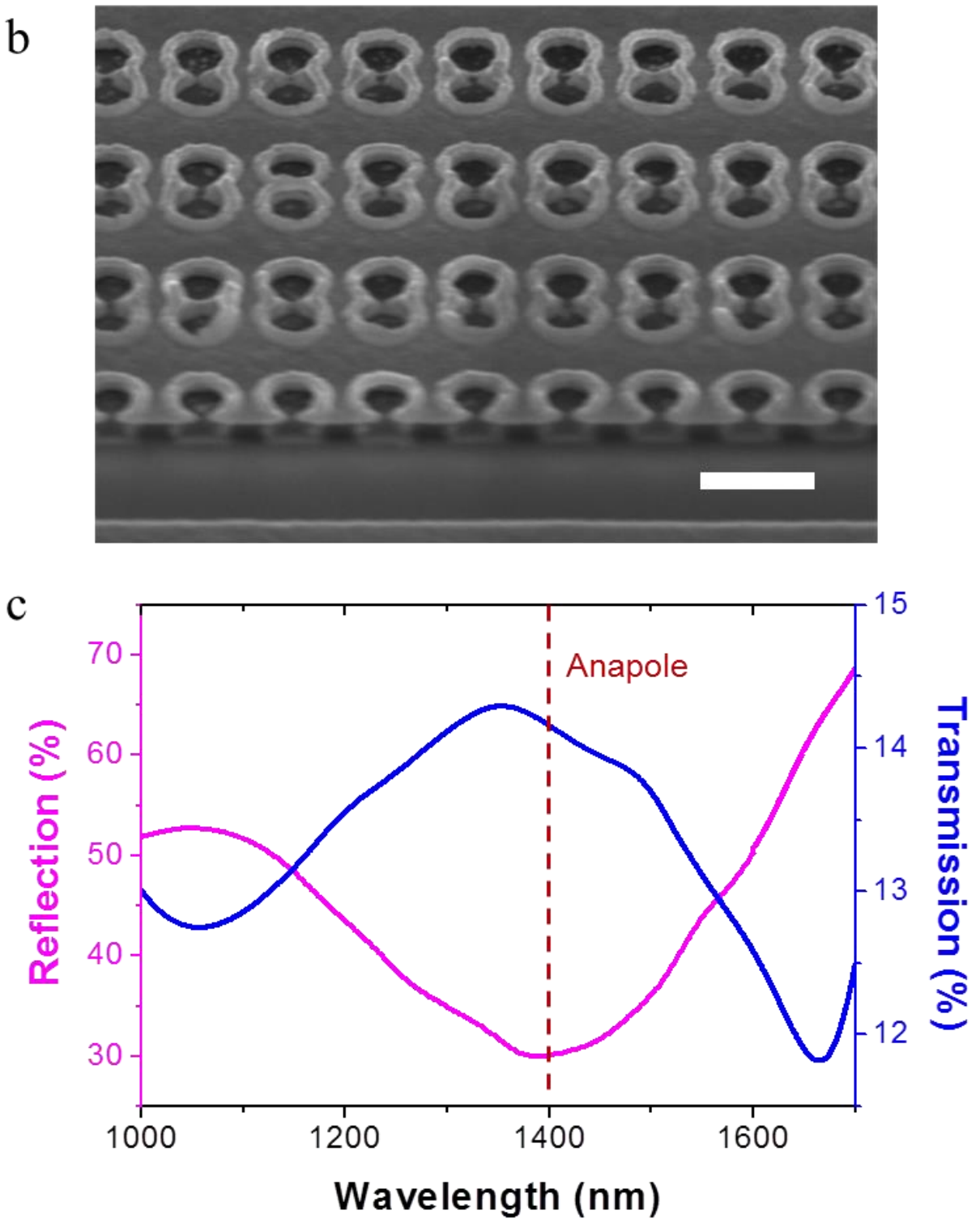

Figure 6 


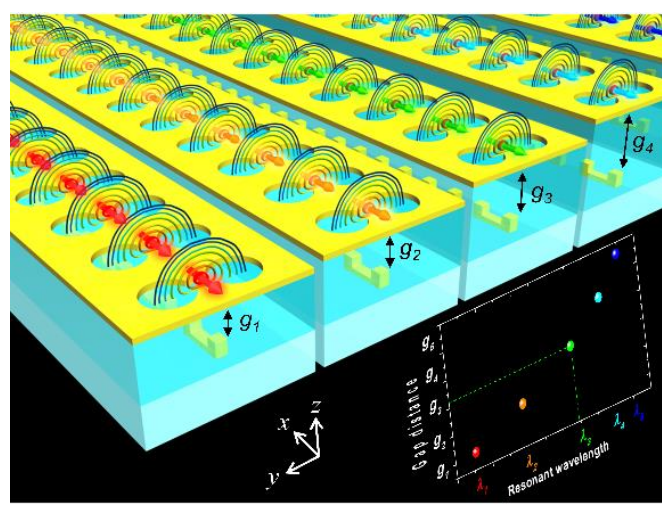

TOC 